Published in final edited form as:

Clin Lab Med. 2017 June ; 37(2): 303-315. doi:10.1016/j.cll.2017.01.005.

\title{
Carbapenem-Resistant Enterobacteriaceae
}

\author{
Alina lovleva, MD and \\ Division of Infectious Diseases, University of Pittsburgh School of Medicine, Falk Medical Building \\ Suite 3A, 3601 Fifth Avenue, Pittsburgh, PA 15213 \\ Yohei Doi, MD, PhD \\ Division of Infectious Diseases, University of Pittsburgh School of Medicine, S829 Scaife Hall, \\ 3550 Terrace Street, Pittsburgh, PA 15261
}

\section{Synopsis}

Carbapenem-resistant Enterobacteriaceae (CRE) have emerged as a major threat to modern medicine. Commonly used antibiotics are generally inactive against CRE. Therefore, timely detection of CRE in the clinical laboratory is of paramount importance. Among CRE, those producing carbapenem-hydrolyzing $\beta$-lactamase enzymes (carbapenemase-producing Enterobacteriaceae; $\mathrm{CPE}$ ) are particularly of concern since they tend to spread among patients, and treatment of active infection is difficult. The carbapenemase groups most commonly encountered include KPC, NDM and OXA-48, with KPC overrepresented in the United States. Various phenotypic and genetic tests have been proposed and validated for rapid detection of CPE. Phenotypic tests detect carbapenemase activity either by inhibiting their activity with specific inhibitors of carbapenemases, or by detecting hydrolysis of carbapenems by carbapenemases. Genetic tests mostly depend on nucleic acid detection and amplification. Treatment options for CRE infections are limited and typically include combinations of polymyxins, tigecycline, aminoglycosides, or carbapenems, but newer agents with activity against CRE and better safety profiles are starting to become available and will likely emerge as the preferred therapy for the treatment of CRE infections in the near future.

\section{Keywords}

carbapenemase; KPC; rapid diagnosis

\section{Introduction}

Carbapenem antibiotics are generally considered to be the most potent group of antimicrobial agents with proven efficacy in the treatment of patients with severe bacterial

\footnotetext{
Correspondence to: Yohei Doi.

Transparency declarations

Y.D. has served on advisory boards for Meiji, Achaogen, Allergan, Curetis, and has received research funding from The Medicines Company for a study unrelated to this work. A.I declares no conflicts of interest.

Publisher's Disclaimer: This is a PDF file of an unedited manuscript that has been accepted for publication. As a service to our customers we are providing this early version of the manuscript. The manuscript will undergo copyediting, typesetting, and review of the resulting proof before it is published in its final citable form. Please note that during the production process errors may be discovered which could affect the content, and all legal disclaimers that apply to the journal pertain.
} 
infections, including those caused by otherwise antimicrobial-resistant strains. The recent increase in the rates of carbapenem-resistant Enterobacteriaceae (CRE) among healthcareassociated Enterobacteriaceae species, in particular Klebsiella pneumoniae, is therefore a major cause for concern. This surge in CRE is mostly driven by the emergence and spread of carbapenemases, a specific group of $\beta$-lactamases that are capable of hydrolyzing carbapenems. Most strains that produce carbapenemases are resistant to carbapenems, and those that are not demonstrate reduced susceptibility to these agents.

Three groups of carbapenemases - KPC, NDM, and OXA-48 - are currently considered to be the three major $\beta$-lactamases of epidemiological and clinical significance. In the U.S., KPC is by far the most common carbapenemase produced by CRE, ${ }^{1}$ but outbreaks of NDMproducing Enterobacteriaceae have been reported from U.S. hospitals; ;,3 OXA-48-producing Enterobacteriaceae strains have also been reported sporadically. ${ }^{4}$ It is essential that clinical microbiology laboratories be capable of recognizing CRE strains that produce these key groups of carbapenemases and refer them for further testing when appropriate to inform clinicians and infection preventionists. This review is intended to provide clinical microbiologists with an overview of the epidemiology, diagnosis and clinical implications of CRE.

\section{History of CRE}

The discovery and clinical application of antimicrobial agents constitutes one of the greatest public health achievements of the $20^{\text {th }}$ century, drastically reducing mortality from common infectious diseases like pneumonia and diarrheal illnesses. ${ }^{5}$ However, the introduction of every new class of antimicrobial agents has been eclipsed by emergence of bacteria that are resistant to them. $\beta$-lactams, arguably the most successful antimicrobial class used in clinical practice, have not been an exception in this regard. The introduction of ampicillin as an antiGram-negative aminopenicillin in the 1960s was quickly followed by the spread of Escherichia coli that produce TEM-1 $\beta$-lactamase, which is capable of hydrolyzing ampicillin. ${ }^{6}$ To counter this, various oxyimino-cephalosporins (e.g., cefotaxime, ceftazidime) were introduced in the 1980s, which were by design stable against hydrolysis by TEM-1 or SHV-1 ( $\beta$-lactamase naturally produced by Klebsiella pneumoniae and conferring ampicillin resistance). However, Enterobacteriaceae countered them several years later by generating variants of TEM-1 and SHV-1, which have extended the spectrum of hydrolysis to include not only aminopenicillins but also oxyimino-cephalosporins (thus the name extended-spectrum $\beta$-lactamases, or ESBLs). ${ }^{6}$ ESBL producers were resistant to oxyimino-cephalosporins. Carbapenems were then introduced to clinics in the late 1980s and proved highly efficacious in the treatment of ESBL-producing $K$. pneumoniae infections. $^{7}$

Unfortunately, even carbapenems were not immune to the remarkable ability of Enterobacteriaceae to adapt to selective pressure. In the early 1990s, Enterobacteriaceae with resistance to carbapenems (carbapenem-resistant Enterobacteriaceae, or CRE) emerged in Japan, followed by neighboring countries. ${ }^{8}$ These strains produced metallo- $\beta$-lactamase (MBL) IMP-1, which was capable of hydrolyzing carbapenems and was encoded on plasmids that could transfer from one species to another. This was followed by discovery of 
VIM-1, another acquired MBL, which was initially identified from Pseudomonas aeruginosa in Italy and subsequently found in Enterobacteriaceae. ${ }^{9}$ In the United States, a $K$. pneumoniae strain with resistance to carbapenems was identified in 1996. This strain produced a novel carbapenemase, which was later coined KPC for Klebsiella pneumoniae carbapenemase. ${ }^{10}$ This KPC gene is encoded on a transferable plasmid and is capable of hydrolyzing both oxyimino-cephalosporins and carbapenems efficiently. It became apparent by the early 2000s that KPC-producing $K$. pneumoniae was rapidly becoming endemic at hospitals in parts of New York City. ${ }^{11,12}$ Since then, KPC-producing $K$. pneumoniae has spread across the continental U.S. and many other countries worldwide causing both outbreaks and endemicity in certain regions. ${ }^{1}$ In parallel to the expansion of KPC in the U.S. and elsewhere, another group of carbapenemases, OXA-48, emerged and spread mostly in $K$. pneumoniae in the Mediterranean countries in the 2000s. ${ }^{13,14}$ More recently, a novel group of MBL, NDM (New Delhi metallo- $\beta$-lactamase), was identified and reported in carbapenem-resistant $K$. pneumoniae and E. coli in a patient who had traveled from India in 2009. ${ }^{15}$ NDM-1 has since spread explosively in South Asia and also globally. ${ }^{16,17}$

\section{CRE and CPE}

Both the terms CRE (carbapenem-resistant Enterobacteriaceae) and CPE (carbapenemaseproducing Enterobacteriaceae) appear in the literature and are sometimes used interchangeably. While there is certainly a significant overlap between CRE and CPE, it is important to distinguish them since the former refers to the resistance phenotype, whereas the latter is defined by the mechanism underlying the phenotype. Carbapenemase production is certainly the most prominent mechanism underlying carbapenem resistance in Gramnegative pathogens. Other mechanisms such as overproduction of AmpC or production of extended-spectrum $\beta$-lactamase can function together with outer membrane protein deficiency and overproduction of certain efflux pumps to confer carbapenem resistance, especially in species such as Enterobacter spp. On the other hand, carbapenemase production usually results in clinically relevant levels of carbapenem resistance, but on occasion may only yield reduced susceptibility that does not reach the susceptibility breakpoints. While CRE poses challenges with treatment in general, CPE is considered to be a more significant concern for both infection prevention and treatment since carbapenemase genes are mostly carried on plasmids that have the ability to transfer between bacterial species. Consequently, outbreaks due to CPE are commonly reported, and CPE infections are associated with high mortality. ${ }^{12,18}$ Rapid detection of CPE facilitates timely implementation of appropriate infection prevention measures, in addition to informing clinicians who must decide on treatment regimens for CRE and CPE infections.

\section{Spectrum of resistance conferred by carbapenemases}

The three major groups of carbapenemases belong to separate molecular classes as defined by Ambler ${ }^{19}$ : KPC belongs to class A, NDM to class B and OXA-48 to class D (Table 1). KPC utilizes a serine residue for its activity and has a very broad spectrum of substrates including penicillins, cephalosporins, classic $\beta$-lactamase inhibitors (clavulanic acid, sulbactam and tazobactam), aztreonam and carbapenems. Its activity is only minimally inhibited by clavulanic acid but is inhibited well by boronic acid compounds. ${ }^{20} \mathrm{MBLs}$ 
including NDM are metalloenzymes that possess zinc in the active site. The spectrum of hydrolysis is similar to that of KPC but spares aztreonam. Therefore, resistance to carbapenems and susceptibility to aztreonam is suggestive of MBL production; however, coproduction of ESBL is common in clinical strains, which make them also resistant to aztreonam. ${ }^{21}$ OXA-48 is a serine $\beta$-lactamase like KPC, but has a unique spectrum of activity that includes penicillins and carbapenems but spares cephalosporins and aztreonam. In addition, its activity against carbapenems is not as robust as that of KPC and MBLs. For this reason, detection of OXA-48-producing Enterobacteriaceae based on susceptibility phenotype is more challenging and requires attention to subtle irregularities, such as reduced susceptibility to carbapenems (e.g. ertapenem MIC of $\searrow 0.5 \mathrm{mg} / \mathrm{L}$, or imipenem or meropenem MIC of $\geq 1 \mathrm{mg} / \mathrm{L}$ ), which may not meet the current criteria for intermediate resistance or resistance. ${ }^{22}$

\section{Epidemiology of CRE}

The prevalence of CRE as well as the types of common carbapenemases is highly dependent on geography, but the highest rates are seen in $K$. pneumoniae over other species of the family Enterobacteriaceae across regions. It is also seen almost exclusively in healthcareassociated infections, with the exception of the Indian subcontinent where communityassociated CRE infections due to NDM-producing strains have been reported. ${ }^{23}$ Countries known to have high overall rates of CRE include Greece, Italy, Brazil and China, followed by several other countries including the U.S. and Colombia. Countries in the Indian subcontinent have a high burden of CRE that is driven by the spread of NDM-producing strains, but prevalence data are scarce. CRE rates among $K$. pneumoniae are as high as $62 \%$ in Greece and $33 \%$ in Italy. ${ }^{24}$ In the United States, $11 \%$ of $K$. pneumoniae causing healthcare-associated infections were resistant to carbapenems in 2014, representing a modest decline from 2013 (13\%). ${ }^{25}$ The majority of CRE cases are concentrated in the MidAtlantic (New York, New Jersey and Pennsylvania), the Midwest (Michigan, Illinois), and the Southeast (Florida). ${ }^{26}$

Globally, KPC is the most commonly observed carbapenemase in Enterobacteriaceae, and KPC-2 and KPC- 3 are by far the most predominant alleles. ${ }^{1}$ Countries with high prevalence of KPC-producing K. pneumoniae include the U.S., as well as some countries in South America (Brazil, Colombia), Europe (Italy, Greece), and East Asia (China in particular). Most CREs in the U.S. produce KPC carbapenemases. ${ }^{27} \mathrm{KPC}$-producing $K$. pneumoniae is characterized by its clonality, in that the majority of the strains circulating globally belong to clonal complex (CC) 258 as defined by multilocus sequence typing (MLST). This suggests that CC258 acquired the KPC gene at an early stage in this epidemic and spread successfully. ${ }^{28}$ The most common sequence type (ST) is ST258 in the U.S., but other CC258 STs such as ST11, ST340, ST437 and ST512 predominate in countries outside the U.S. ${ }^{29}$

NDM is the most common carbapenemase in the Indian subcontinent (India, Pakistan, Bangladesh), and also possibly in some of the Balkan nations. ${ }^{30}$ While many NDM variants have been reported, NDM-1 continues to be the most frequent allele in this group of carbapenemases. NDM-producing Enterobacteriaceae are also increasingly reported from China, but the overall epidemiology there is yet unclear since their prevalence is typically 
reported from single hospitals or locales. ${ }^{31}$ Organisms producing NDM carbapenemases have been sporadic in the U.S. and are mostly associated with direct importation from the Indian subcontinent, ${ }^{32}$ but some locally acquired cases may be emerging. ${ }^{33}$ Unlike with KPC-producing $K$. pneumoniae where CC258 is the predominant clonal lineage, the spread of NDM-producing Enterobacteriaceae is explained by horizontal transfer of epidemic broad host-range plasmids carrying the NDM gene. ${ }^{34}$

OXA-48 is the third globally distributed group of carbapenemases, which comprises the canonical OXA-48 as well as its variants OXA-181 and OXA-232. ${ }^{34}$ Outbreak reports of OXA-48-producing Enterobacteriaceae are concentrated in European and Mediterranean countries including North Africa, whereas sporadic cases have been identified in the U.S., often in association with recent travel to India., ${ }^{4,22}$

\section{Detection of carbapenemases}

Several approaches have been developed to identify the presence of carbapenemases in Enterobacteriaceae. These include phenotypic and genotypic (nuclear amplification-based) tests. Below is a brief overview of currently available methods.

\section{Phenotypic tests}

\section{Modified Hodge test}

In the modified Hodge test (MHT), a tenth dilution of McFarland 0.5 suspension of E. coli ATCC 25922 is used to evenly inoculate a Mueller-Hinton agar plate, and a10- $\mu \mathrm{g}$ imipenem disk is placed in the center of the plate. The suspected CPE strain is then streaked from the edge of the disk to the periphery of the plate to form a straight line of thick inoculum. After overnight incubation, if a carbapenemase is produced by the test strain, imipenem in the agar plate is hydrolyzed, allowing the susceptible E. coli in the background to grow in towards the disk, creating a cloverleaf-like appearance. ${ }^{35}$ The sensitivity and specificity of the MHT was shown to be excellent in detecting KPC-producing strains earlier in the CRE epidemic, making it one of the recommended confirmation tests of CPE by the Clinical and Laboratory Standards Institute (CLSI). However, the technique is somewhat operator dependent, and occasional false positive results produced by AmpC-producing strains make interpretation difficult. In addition, the MHT lacks the desired sensitivity in detecting MBL-producing strains. ${ }^{36}$ Nonetheless, the MHT does not require any special supplies and detects KPC producers well, and therefore can still be useful in settings where resources are limited and $\mathrm{KPC}$ is the predominant carbapenemase.

\section{Carbapenem inactivation method}

The Carbapenem Inactivation Method (CIM) is a more recently described test to detect CPE. ${ }^{37}$ With the CIM, a 10- $\mu$ g meropenem disk is first incubated in a suspension of the test strain at $35^{\circ} \mathrm{C}$ for 2 hours. After incubation, the meropenem disk is removed from the solution and placed on a Mueller Hinton agar plate inoculated with susceptible E. coli as with the MHT. If carbapenemase is produced, the meropenem in the disk would have been inactivated during the initial incubation, allowing for uninhibited growth of E. coli, making it appear as if the susceptible $E$. coli is resistant. If no carbapenemase is produced, a clear 
inhibition zone is formed. This method has been validated against Enterobacteriaceae strains producing KPC, OXA-48, NDM, VIM and IMP carbapenemases, with sensitivity reaching $99 \% .{ }^{38}$ It is low-cost and does not require any special supplies or skills. For these reasons, it is now endorsed as one of the first-line confirmation tests of CPE by the CLSI.

\section{EDTA inhibition test}

The ability of etylenediaminetetraacetic acid (EDTA) to chelate metal ions makes it a useful compound in detecting MBL, as the activity of these enzymes is dependent on zinc ions. ${ }^{39}$ With the EDTA inhibition test, an EDTA solution is added to a carbapenem disk, which is then placed on a plate inoculated with the strain of interest. If MBL is present, a larger zone of inhibition will form around the EDTA-carbapenem disk compared with the carbapenem disk without EDTA (control). ${ }^{40}$ The same principle can be applied to other metal chelators such as sodium mercaptoacetic acid. ${ }^{41}$

\section{Boronic acid inhibition test}

Boronic acid compounds have been known to be excellent inhibitors of class $C \beta$ lactamases ${ }^{42}$ and have been utilized for detection of this group of enzymes,${ }^{43}$ but more recently have been recognized as an excellent inhibitor of class A carbapenemases including KPC. This property has been applied in detecting production of KPC carbapenemase. ${ }^{44}$ With this test, 300 or $400 \mu \mathrm{g}$ of 2-aminophenyl boronic acid is applied to an ertapenem or meropenem disk. An increase in the zone of inhibition of $\geq 5 \mathrm{~mm}$ compared to the ertapenem or meropenem disk alone (control) is indicative of KPC production.

\section{Carba NP test}

The Carba NP test, originally developed by the group led by Drs. Patrice Nordmann and Laurent Poirel, is a rapid colorimetric test, which is based on detection of $\mathrm{pH}$ changes that accompany hydrolysis of imipenem by carbapenemases. ${ }^{45}$ In the original Carba NP test, the test strain first undergoes a lysis step, then the lysate is added to a solution consisting of imipenem monohydrate, phenol red and $\mathrm{ZnSO}_{4}$ (initial $\mathrm{pH}$ of 7.8). This final solution is then incubated at $37^{\circ} \mathrm{C}$ for up to 2 hours. If carbapenemase is produced, the imipenem is hydrolyzed, resulting in reduction of the $\mathrm{pH}$ and color change from red to orange or yellow (Figure 1). While 2 hours of incubation is recommended, KPC producers can be detected in as little as 10 minutes. The Carba NP test is highly sensitive in detecting KPC and most MBL-producing strains. However, it may have difficulty identifying activity of relatively weak carbapenemases such as OXA-48 and GES-5, and also some mucoid strains. ${ }^{46}$ The Carba NP test is one of the methods endorsed by the CLSI for the detection of carbapenemase production.

\section{MALDI-TOF}

Matrix-assisted laser desorption/ionisation time-of-flight mass spectrometry (MALDI-TOF MS) assays to detect carbapenemase activity have been developed by many investigators. ${ }^{47}$ Instruments that are currently used in clinical microbiology laboratories, such as Bruker Microflex, can be used for this purpose. While the details vary, a colony of the test strain is suspended in a solution containing a carbapenem and incubated at $37^{\circ} \mathrm{C}$ for a period ranging 
from 15 minutes to as long as several hours. A small fraction of this solution is loaded onto a target plate with a matrix after which mass spectra are acquired. For example, imipenem has an $\mathrm{m} / \mathrm{z}$ of 300 , and its dominant metabolite has an $\mathrm{m} / \mathrm{z}$ of 254 . Therefore, predominant detection of the latter peak relative to that of the intact imipenem indicates production of carbapenemase. Sensitivity and specificity of nearly $100 \%$ have been reported, with a turnaround time as short as 30 minutes. ${ }^{48,49}$ However, false negative results with OXA-48producing strains and slime-producing strains due to interactions with polysaccharide have been reported. While most published protocols use pure culture, successful use of bacteria collected directly from positive blood cultures have also been reported. ${ }^{50}$ While the protocol and analysis are yet to be standardized, use of MALDI-TOF MS appears to be a practical option for laboratories that have access to this technology.

\section{Molecular tests}

\section{Conventional PCR/real-time PCR}

Detection of carbapenemase genes can be achieved by conventional or real-time PCR. Multiplex PCR assays for the common carbapenemase genes have been developed and can be utilized in settings where such assays are available.

\section{Verigene}

Verigene Gram-negative blood culture assay (Luminex Corporation) is a non-amplified test that relies on nucleic acid extraction from positive blood cultures, followed by microarraybased detection using capture and detection probes. The test requires approximately 5 minutes of hands-on time and 2 hours to run. In addition to identification of 8 Gram-negative species, it is capable of detecting bla $a_{\mathrm{CTX}-\mathrm{M}}$, bla $_{\mathrm{KPC}}$, bla $_{\mathrm{NDM}}$, bla $_{\mathrm{VIM}}, b l a_{\mathrm{IMP}}$, and bla $a_{\mathrm{OXA}}$ (including $b a_{\text {OXA-48 }}$ ). The positive percentage agreement in the multicenter validation study was as follows: bla $a_{\mathrm{CTX}-\mathrm{M}}, 98.9 \%$; bla $_{\mathrm{KPC}}, 100 \%$; bla $_{\mathrm{NDM}}, 96.2 \%$; bla $\mathrm{OXX}_{\mathrm{XA}}, 94.3 \%$; bla $_{\mathrm{VIM}}$, $100 \%$; and bla IMP $_{\mathrm{IMP}}, 100 \% .^{51}$ The materials cost is approximately $\$ 60$ to $\$ 80$ per test. ${ }^{52}$

\section{BioFire FilmArray}

The FilmArray Blood Culture Identification Panel (bioMérieux) is an automated multiplex PCR system that is carried out in a closed, disposable, single-use pouch. ${ }^{53}$ It is primarily geared towards rapid identification of bacteremia and fungemia directly from positive blood culture bottles, but it is also able to detect the presence of bla $a_{\mathrm{KPC}}$ along with mec $A$ and $\operatorname{van} A / v a n B .{ }^{54}$ The hands-on time for sample preparation is 2 to 3 minutes, and the results are available in about 1 hour. Sensitivity and specificity of $100 \%$ have been reported for the detection of bla $a_{\mathrm{KPC}}$. However, a test on FilmArray costs over $\$ 100$, which limits the specific utility of this platform in detection bla $a_{\mathrm{KPC}}$ in low to moderate prevalence settings. In addition, a negative test may not be informative in locales where other types of carbapenemases are found.

\section{Xpert}

The Xpert Carba-R (Cephid) detects bla $a_{\mathrm{KPC}}$, bla $_{\mathrm{NDM}}$, bla $_{\mathrm{VIM}}$, bla $_{\mathrm{IMP}}$, and blaOXA-48. The system has been approved for use in rectal swab specimens in the U.S. The test requires hands-on time of 1 minute and run time of less than an hour, and can be run on an existing 
GeneXpert platform. The sensitivity, specificity, and positive and negative predictive values of the Xpert Carba-R assay compared to those of the reference culture and sequencing results have been reported to be $96.6 \%, 98.6 \%, 95.3 \%$, and $99.0 \%$, respectively. ${ }^{55}$ Since Xpert Carba-R only detects the presence of carbapenemase genes, information regarding the species carrying them (e.g., K. pneumoniae) is not available.

\section{Genome and metagenome sequencing}

Conceptually, whole genome sequencing (WGS) is an attractive method for the detection of carbapenemase genes since it can identify known as well as yet unknown genes that encode carbapenemases. An added advantage is that it will also yield information regarding species, clades, and any resistance genes other than carbapenemase genes. The cost of WGS has declined precipitously over the last decade, to a point that application in clinical microbiology laboratories is in sight. However, there are several hurdles that need to be overcome before the technology can be incorporated into routine clinical microbiology, including turnaround time and data management. ${ }^{56}$

Metagenome sequencing uses similar technology as WGS, but instead of using pure culture DNA as the starting material, it uses DNA extracted directly from biological specimens such as sputum. Because of the high cost and complexity of the data analysis, it is currently evaluated for the diagnosis of infections for which currently available diagnostic methods do not suffice. This technique will be able to detect carbapenemase genes present in the specimens tested, however it will not provide information on the species of the strain(s) producing carbapenemase.

\section{Treatment of CRE infections}

\section{Currently available therapy}

CRE strains are usually resistant to all $\beta$-lactam agents, including carbapenems and $\beta$ lactamase inhibitor combinations (with a notable exception of ceftazidime-avibactam). The majority of these strains are still susceptible to polymyxins (colistin and polymyxin B) and tigecycline. Additionally some KPC-producing and OXA-48-producing strains are susceptible to aminoglycosides (gentamicin or amikacin). NDM-producing strains are typically resistant to all aminoglycosides.

Since polymyxins and tigecycline do not have the most desirable pharmacokinetic properties, mortality of patients with invasive CRE infections is high when treated with a single active agent (monotherapy). Therefore, treatment with two or more active agents (combination therapy) is routinely considered. ${ }^{57}$ There are no published randomized clinical trials that specifically address treatment of CRE infections at this time, and the best available data are retrospective cohort studies examining treatment and mortality outcome of patients with invasive CRE infections. In a systematic review of the clinical outcome of CRE infections in 889 patients, most of which were due to KPC-producing K. pneumoniae, 441 received combination therapy, 346 received monotherapy, and 102 received inactive therapy ${ }^{58}$ The mortality rates of monotherapy were $40.1 \%$ for carbapenem, $41.1 \%$ for tigecycline and $42.8 \%$ for colistin, whereas the mortality for inactive therapy was $46.1 \%$. In 
contrast, the mortality rates for combination therapy were $30.7 \%$ for carbapenem-sparing combinations and $18.8 \%$ for carbapenem-containing combinations. Based on these data, carbapenem-containing regimens (such as meropenem combined with colistin, tigecycline, or gentamicin) are frequently employed for the treatment of invasive CRE infections. It has also been suggested that when the KPC-producing $K$. pneumoniae develops resistance to colistin but remains susceptible to gentamicin, gentamicin-containing regimens may be associated with lower mortality. ${ }^{59}$

\section{New agents in development}

There are multiple new agents that are in clinical development and have activity against CRE. They can be divided into $\beta$-lactam/ $\beta$-lactamase inhibitor combinations and others. Among them, ceftazidime-avibactam is already available for clinical use in the U.S. since 2015. Avibactam is a novel $\beta$-lactamase inhibitor that inhibits KPC, ESBL, AmpC and OXA-48. As a result, the ceftazidime-avibactam combination is active against most KPCand OXA-48-producing strains. However, it is not active against MBL-producing strains, including those producing NDM. ${ }^{60}$ While efficacy data are yet scarce, a 30-day mortality of $24 \%$ has been reported in a case series of CRE infections treated with ceftazidimeavibactam, which is reasonably low when considering the ill patient population. ${ }^{61}$ However, resistance to ceftazidime-avibactam, either intrinsic or acquired, has already been reported. This raises the question whether this agent can be used as monotherapy or should be used in the context of combination therapy to prevent emergence of resistance. ${ }^{33,61}$ Two other $\beta$ lactam/ $\beta$-lactamase inhibitor combinations are in late-stage clinical development, including meropenem-vaborbactam and imipenem-cilastatin-relebactam. While the structures of the inhibitors and the partner agents differ, the overall spectrum of activity of these agents is similar to that of ceftazidime-avibactam. ${ }^{62}$ Agents from other classes in late-stage clinical development include plazomicin, eravacycline and cefiderocol. Plazomicin is a new aminoglycoside derived from sisomicin and designed to resist most aminoglycosidemodifying enzymes. It is active against most Enterobacteriaceae strains, including KPCproducing strains, but activity against NDM-producing strains is limited due to frequent coproduction of $16 \mathrm{~S}$ ribosomal RNA methyltransferase which confers resistance to aminoglycosides including plazomicin. Eravacycline is a novel fluorocycline of the tetracycline class with broad activity against Gram-negative bacteria including CRE. Its spectrum of activity is similar to that of tigecycline, but it may have an advantage in terms of in vitro activity, pharmacokinetics, and tolerability. ${ }^{62}$ Finally, cefiderocol (S-649266) is a novel siderophore cephalosporin with a catechol moiety that demonstrates in vitro activity against CRE, including both KPC-producing and MBL-producing Enterobacteriaceae. ${ }^{63}$ The availability of these new anti-CRE agents is expected to transform treatment approaches for invasive CRE infections in the very near future.

\section{Conclusion}

CRE has spread in healthcare settings worldwide in the last decade. CRE infections are notoriously difficult to treat and associated with high mortality because of limited treatment options. Timely and accurate detection of CRE, in particular those producing carbapenemase, or CPE, is therefore of paramount importance both to guide clinicians and 
to inform infection preventionists. Several phenotypic and genetic methods are available for rapid detection of carbapenemase production, many of which, especially the phenotypic ones, can be implemented in regular clinical microbiology laboratories. Treatment of CRE infections usually consist of combination of active antimicrobial agents, but newer agents with improved activity and safety are in clinical development, some of which we expect to become available for clinical use in the near future.

\section{Acknowledgments}

Funding

The effort of Y.D. was supported by research grants from the National Institutes of Health (R01AI104895, R21AI123747).

\section{References}

1. Munoz-Price LS, Poirel L, Bonomo RA, et al. Clinical epidemiology of the global expansion of Klebsiella pneumoniae carbapenemases. Lancet Infect Dis. 2013; 13:785-796. [PubMed: 23969216]

2. Epson EE, Pisney LM, Wendt JM, et al. Carbapenem-resistant Klebsiella pneumoniae producing New Delhi metallo- $\beta$-lactamase at an acute care hospital, Colorado, 2012. Infect Control Hosp Epidemiol. 2014; 35:390-397. [PubMed: 24602944]

3. Epstein L, Hunter JC, Arwady MA, et al. New Delhi metallo- $\beta$-lactamase-producing carbapenemresistant Escherichia coli associated with exposure to duodenoscopes. JAMA. 2014; 312:14471455. [PubMed: 25291580]

4. Lyman M, Walters M, Lonsway D, Rasheed K, Limbago B, Kallen A. Notes from the field: Carbapenem-resistant Enterobacteriaceae producing OXA-48-like carbapenemases--United States, 2010-2015. MMWR Morb Mortal Wkly Rep. 2015; 64:1315-1316. [PubMed: 26633574]

5. Centers for Disease C, Prevention. Ten great public health achievements--United States, 1900-1999. MMWR Morb Mortal Wkly Rep. 1999; 48:241-243. [PubMed: 10220250]

6. Paterson DL, Bonomo RA. Extended-spectrum $\beta$-lactamases: a clinical update. Clin Microbiol Rev. 2005; 18:657-686. [PubMed: 16223952]

7. Paterson DL, Ko WC, Von Gottberg A, et al. Antibiotic therapy for Klebsiella pneumoniae bacteremia: implications of production of extended-spectrum $\beta$-lactamases. Clin Infect Dis. 2004; 39:31-37. [PubMed: 15206050]

8. Osano E, Arakawa Y, Wacharotayankun R, et al. Molecular characterization of an enterobacterial metallo $\beta$-lactamase found in a clinical isolate of Serratia marcescens that shows imipenem resistance. Antimicrob Agents Chemother. 1994; 38:71-78. [PubMed: 8141584]

9. Lauretti L, Riccio ML, Mazzariol A, et al. Cloning and characterization of bla $\mathrm{VIM}$, a new integronborne metallo- $\beta$-lactamase gene from a Pseudomonas aeruginosa clinical isolate. Antimicrob Agents Chemother. 1999; 43:1584-1590. [PubMed: 10390207]

10. Yigit H, Queenan AM, Anderson GJ, et al. Novel carbapenem-hydrolyzing $\beta$-lactamase, KPC-1, from a carbapenem-resistant strain of Klebsiella pneumoniae. Antimicrob Agents Chemother. 2001; 45:1151-1161. [PubMed: 11257029]

11. Bradford PA, Bratu S, Urban C, et al. Emergence of carbapenem-resistant Klebsiella species possessing the class A carbapenem-hydrolyzing KPC-2 and inhibitor-resistant TEM-30 $\beta$ lactamases in New York City. Clin Infect Dis. 2004; 39:55-60. [PubMed: 15206053]

12. Bratu S, Landman D, Haag R, et al. Rapid spread of carbapenem-resistant Klebsiella pneumoniae in New York City: a new threat to our antibiotic armamentarium. Arch Intern Med. 2005; 165:1430-1435. [PubMed: 15983294]

13. Poirel L, Heritier C, Tolun V, Nordmann P. Emergence of oxacillinase-mediated resistance to imipenem in Klebsiella pneumoniae. Antimicrob Agents Chemother. 2004; 48:15-22. [PubMed: 14693513] 
14. Potron A, Poirel L, Rondinaud E, Nordmann P. Intercontinental spread of OXA-48 $\beta$-lactamaseproducing Enterobacteriaceae over a 11-year period, 2001 to 2011. Euro Surveill. 2013:18.

15. Yong D, Toleman MA, Giske CG, et al. Characterization of a new metallo- $\beta$-lactamase gene, bla $_{\mathrm{NDM}-1}$, and a novel erythromycin esterase gene carried on a unique genetic structure in Klebsiella pneumoniae sequence type 14 from India. Antimicrob Agents Chemother. 2009; 53:5046-5054. [PubMed: 19770275]

16. Kumarasamy KK, Toleman MA, Walsh TR, et al. Emergence of a new antibiotic resistance mechanism in India, Pakistan, and the UK: a molecular, biological, and epidemiological study. Lancet Infect Dis. 2010; 10:597-602. [PubMed: 20705517]

17. Nordmann P, Poirel L, Walsh TR, Livermore DM. The emerging NDM carbapenemases. Trends Microbiol. 2011; 19:588-595. [PubMed: 22078325]

18. Tumbarello M, Viale P, Viscoli C, et al. Predictors of mortality in bloodstream infections caused by Klebsiella pneumoniae carbapenemase-producing $K$. pneumoniae: importance of combination therapy. Clin Infect Dis. 2012; 55:943-950. [PubMed: 22752516]

19. Ambler RP. The structure of $\beta$-lactamases. Philos Trans R Soc Lond B Biol Sci. 1980; 289:321331. [PubMed: 6109327]

20. Ke W, Bethel CR, Papp-Wallace KM, et al. Crystal structures of KPC-2 $\beta$-lactamase in complex with 3-nitrophenyl boronic acid and the penam sulfone PSR-3-226. Antimicrob Agents Chemother. 2012; 56:2713-2718. [PubMed: 22330909]

21. Bonomo RA. New Delhi metallo- $\beta$-lactamase and multidrug resistance: a global SOS? Clin Infect Dis. 2011; 52:485-487. [PubMed: 21258101]

22. Poirel L, Potron A, Nordmann P. OXA-48-like carbapenemases: the phantom menace. J Antimicrob Chemother. 2012; 67:1597-1606. [PubMed: 22499996]

23. Borah VV, Saikia KK, Chandra P, Hazarika NK, Chakravarty R. New Delhi metallo- $\beta$-lactamase and extended spectrum $\beta$-lactamases co-producing isolates are high in community-acquired urinary infections in Assam as detected by a novel multiplex polymerase chain reaction assay. Indian J Med Microbiol. 2016; 34:173-182. [PubMed: 27080768]

24. European Centre for Disease Prevention and Control. Antimicrobial resistance surveillance in Europe, 2014. 2015. http://ecdc.europa.eu/en/publications/Publications/antimicrobial-resistanceeurope-2014.pdf

25. Weiner LM, Webb AK, Limbago B, et al. Antimicrobial-resistant pathogens associated with healthcare-associated infections: Summary of data reported to the National Healthcare Safety Network at the Centers for Disease Control and Prevention, 2011-2014. Infect Control Hosp Epidemiol. 2016; 37:1288-1301. [PubMed: 27573805]

26. The Center for Disease Dynamics Economics and Policy. ResistanceMap. 2016. http:// resistancemap.cddep.org/

27. Castanheira M, Farrell SE, Krause KM, Jones RN, Sader HS. Contemporary diversity of $\beta$ lactamases among Enterobacteriaceae in the nine U.S. census regions and ceftazidime-avibactam activity tested against isolates producing the most prevalent $\beta$-lactamase groups. Antimicrob Agents Chemother. 2014; 58:833-838. [PubMed: 24247134]

28. Bowers JR, Kitchel B, Driebe EM, et al. Genomic analysis of the emergence and rapid global dissemination of the clonal group 258 Klebsiella pneumoniae pandemic. PLoS One. 2015; 10:e0133727. [PubMed: 26196384]

29. Chen L, Mathema B, Chavda KD, DeLeo FR, Bonomo RA, Kreiswirth BN. Carbapenemaseproducing Klebsiella pneumoniae: molecular and genetic decoding. Trends Microbiol. 2014; 22:686-696. [PubMed: 25304194]

30. Dortet L, Poirel L, Nordmann P. Worldwide dissemination of the NDM-type carbapenemases in Gram-negative bacteria. Biomed Res Int. 2014; 2014:249856. [PubMed: 24790993]

31. Qin S, Fu Y, Zhang Q, et al. High incidence and endemic spread of NDM-1-positive Enterobacteriaceae in Henan Province, China. Antimicrob Agents Chemother. 2014; 58:42754282. [PubMed: 24777095]

32. Rasheed JK, Kitchel B, Zhu W, et al. New Delhi metallo- $\beta$-lactamase-producing Enterobacteriaceae, United States. Emerg Infect Dis. 2013; 19:870-878. [PubMed: 23731823] 
33. Aitken SL, Tarrand JJ, Deshpande LM, et al. High rates of nonsusceptibility to ceftazidimeavibactam and identification of New Delhi metallo- $\beta$-lactamase production in Enterobacteriaceae bloodstream infections at a major cancer center. Clin Infect Dis. 2016; 63:954-958. [PubMed: 27313265]

34. Pitout JD, Nordmann P, Poirel L. Carbapenemase-producing Klebsiella pneumoniae, a key pathogen set for global nosocomial dominance. Antimicrob Agents Chemother. 2015; 59:58735884. [PubMed: 26169401]

35. Anderson KF, Lonsway DR, Rasheed JK, et al. Evaluation of methods to identify the Klebsiella pneumoniae carbapenemase in Enterobacteriaceae. J Clin Microbiol. 2007; 45:2723-2725. [PubMed: 17581941]

36. Doyle D, Peirano G, Lascols C, Lloyd T, Church DL, Pitout JD. Laboratory detection of Enterobacteriaceae that produce carbapenemases. J Clin Microbiol. 2012; 50:3877-3880. [PubMed: 22993175]

37. van der Zwaluw K, de Haan A, Pluister GN, Bootsma HJ, de Neeling AJ, Schouls LM. The carbapenem inactivation method (CIM), a simple and low-cost alternative for the Carba NP test to assess phenotypic carbapenemase activity in gram-negative rods. PLoS One. 2015; 10:e0123690. [PubMed: 25798828]

38. Tijet N, Patel SN, Melano RG. Detection of carbapenemase activity in Enterobacteriaceae: comparison of the carbapenem inactivation method versus the Carba NP test. J Antimicrob Chemother. 2016; 71:274-276. [PubMed: 26374613]

39. Meini MR, Llarrull LI, Vila AJ. Overcoming differences: The catalytic mechanism of metallo- $\beta$ lactamases. FEBS Lett. 2015; 589:3419-3432. [PubMed: 26297824]

40. Franklin C, Liolios L, Peleg AY. Phenotypic detection of carbapenem-susceptible metallo- $\beta$ lactamase-producing gram-negative bacilli in the clinical laboratory. J Clin Microbiol. 2006; 44:3139-3144. [PubMed: 16954239]

41. Arakawa Y, Shibata N, Shibayama K, et al. Convenient test for screening metallo- $\beta$-lactamaseproducing gram-negative bacteria by using thiol compounds. J Clin Microbiol. 2000; 38:40-43. [PubMed: 10618060]

42. Beesley T, Gascoyne N, Knott-Hunziker V, et al. The inhibition of class C $\beta$-lactamases by boronic acids. Biochem J. 1983; 209:229-233. [PubMed: 6405733]

43. Yagi T, Wachino J, Kurokawa H, et al. Practical methods using boronic acid compounds for identification of class C $\beta$-lactamase-producing Klebsiella pneumoniae and Escherichia coli. J Clin Microbiol. 2005; 43:2551-2558. [PubMed: 15956362]

44. Doi Y, Potoski BA, Adams-Haduch JM, Sidjabat HE, Pasculle AW, Paterson DL. Simple diskbased method for detection of Klebsiella pneumoniae carbapenemase-type $\beta$-lactamase by use of a boronic acid compound. J Clin Microbiol. 2008; 46:4083-4086. [PubMed: 18923005]

45. Nordmann P, Poirel L, Dortet L. Rapid detection of carbapenemase-producing Enterobacteriaceae. Emerg Infect Dis. 2012; 18:1503-1507. [PubMed: 22932472]

46. Tijet N, Boyd D, Patel SN, Mulvey MR, Melano RG. Evaluation of the Carba NP test for rapid detection of carbapenemase-producing Enterobacteriaceae and Pseudomonas aeruginosa. Antimicrob Agents Chemother. 2013; 57:4578-4580. [PubMed: 23817380]

47. Mirande C, Canard I, Buffet Croix Blanche S, et al. Rapid detection of carbapenemase activity: benefits and weaknesses of MALDI-TOF MS. Eur J Clin Microbiol Infect Dis. 2015; 34:22252234. [PubMed: 26337432]

48. Monteferrante CG, Sultan S, Ten Kate MT, et al. Evaluation of different pretreatment protocols to detect accurately clinical carbapenemase-producing Enterobacteriaceae by MALDI-TOF. J Antimicrob Chemother. 2016; 71:2856-2867. [PubMed: 27287232]

49. Lasserre C, De Saint Martin L, Cuzon G, et al. Efficient detection of carbapenemase activity in Enterobacteriaceae by matrix-assisted laser desorption ionization-time of flight mass spectrometry in less than 30 minutes. J Clin Microbiol. 2015; 53:2163-2171. [PubMed: 25926485]

50. Oviano M, Sparbier K, Barba MJ, Kostrzewa M, Bou G. Universal protocol for the rapid automated detection of carbapenem-resistant Gram-negative bacilli directly from blood cultures by matrix-assisted laser desorption/ionisation time-of-flight mass spectrometry (MALDI-TOF/MS). Int J Antimicrob Agents. 2016 
51. Ledeboer NA, Lopansri BK, Dhiman N, et al. Identification of gram-negative bacteria and genetic resistance determinants from positive blood culture broths by use of the Verigene Gram-negative blood culture multiplex microarray-based molecular assay. J Clin Microbiol. 2015; 53:2460-2472. [PubMed: 25994165]

52. Hill JT, Tran KD, Barton KL, Labreche MJ, Sharp SE. Evaluation of the nanosphere Verigene BCGN assay for direct identification of gram-negative bacilli and antibiotic resistance markers from positive blood cultures and potential impact for more-rapid antibiotic interventions. J Clin Microbiol. 2014; 52:3805-3807. [PubMed: 25122857]

53. Salimnia H, Fairfax MR, Lephart PR, et al. Evaluation of the FilmArray blood culture identification panel: Results of a multicenter controlled trial. J Clin Microbiol. 2016; 54:687-698. [PubMed: 26739158]

54. Rand KH, Delano JP. Direct identification of bacteria in positive blood cultures: comparison of two rapid methods, FilmArray and mass spectrometry. Diagn Microbiol Infect Dis. 2014; 79:293-297. [PubMed: 24767683]

55. Tato M, Ruiz-Garbajosa P, Traczewski M, et al. Multisite evaluation of Cepheid Xpert Carba-R assay for detection of carbapenemase-producing organisms in rectal swabs. J Clin Microbiol. 2016; 54:1814-1819. [PubMed: 27122379]

56. Patel R. New developments in clinical bacteriology laboratories. Mayo Clin Proc. 2016; 91:14481459. [PubMed: 27552910]

57. Doi Y, Paterson DL. Carbapenemase-producing Enterobacteriaceae. Semin Respir Crit Care Med. 2015; 36:74-84. [PubMed: 25643272]

58. Tzouvelekis LS, Markogiannakis A, Piperaki E, Souli M, Daikos GL. Treating infections caused by carbapenemase-producing Enterobacteriaceae. Clin Microbiol Infect. 2014; 20:862-872. [PubMed: 24890393]

59. Gonzalez-Padilla M, Torre-Cisneros J, Rivera-Espinar F, et al. Gentamicin therapy for sepsis due to carbapenem-resistant and colistin-resistant Klebsiella pneumoniae. J Antimicrob Chemother. 2015; 70:905-913. [PubMed: 25344809]

60. Falcone M, Paterson D. Spotlight on ceftazidime/avibactam: a new option for MDR Gram-negative infections. J Antimicrob Chemother. 2016; 71:2713-2722. [PubMed: 27432599]

61. Shields RK, Potoski BA, Haidar G, et al. Clinical outcomes, drug toxicity and emergence of ceftazidime-avibactam resistance among patients treated for carbapenem-resistant Enterobacteriaceae infections. Clin Infect Dis. 2016

62. Thaden JT, Pogue JM, Kaye KS. Role of newer and re-emerging older agents in the treatment of infections caused by carbapenem-resistant Enterobacteriaceae. Virulence. 2016:1-14.

63. Kohira N, West J, Ito A, et al. In vitro antimicrobial activity of a siderophore cephalosporin, S-649266, against Enterobacteriaceae clinical isolates, including carbapenem-resistant strains. Antimicrob Agents Chemother. 2016; 60:729-734. [PubMed: 26574013] 


\section{Key Points}

- $\quad$ CRE has spread in healthcare settings worldwide in the last decade.

- $\quad$ Timely and accurate detection of CRE, in particular those producing carbapenemase, or CPE, is of paramount importance both to guide clinicians and to inform infection preventionists.

- Several phenotypic methods are available for rapid detection of carbapenemase production that can be implemented and performed in clinical microbiology laboratories.

- Treatment of CRE infections usually consists of combination of active antimicrobial agents, but newer agents with improved activity and safety profiles are in late-stage clinical development. 


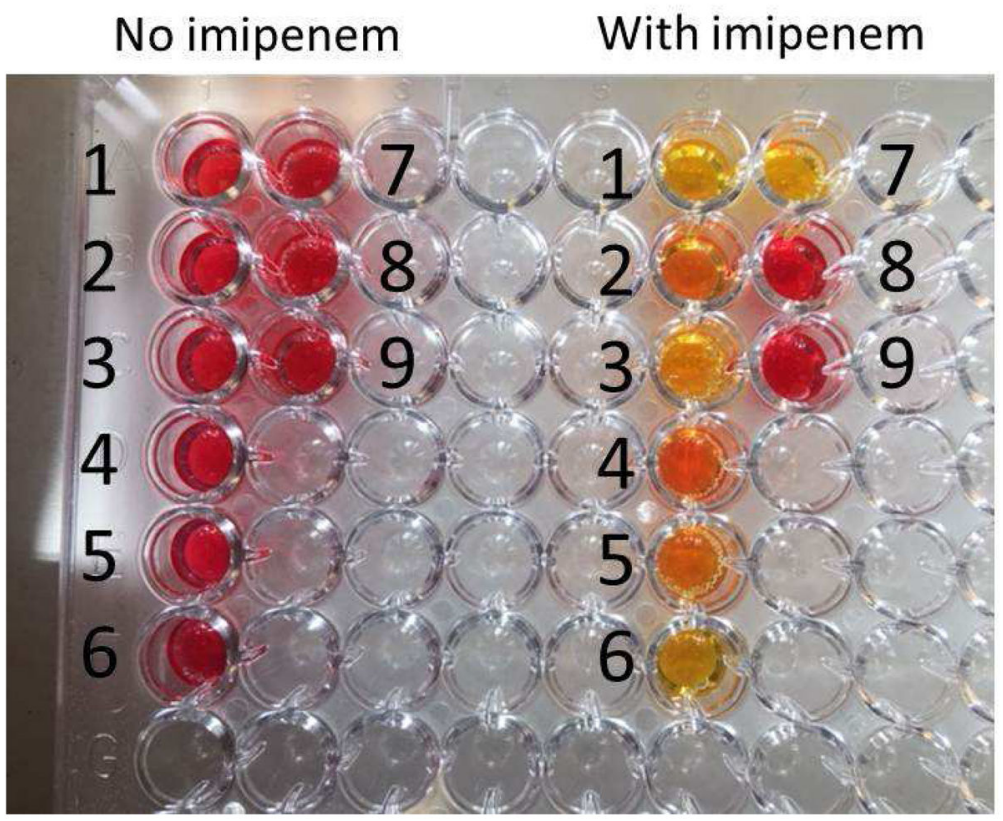

Figure 1.

Example of Carba NP test. Wells 1-7 are carbapenemase-producing strains, wells 8 and 9 are non-carbapanemase-producing strains. Photographed after 1 hour of reaction. 
Table 1

Characteristics of major acquired carbapenemases in Enterobacteriaceae.

\begin{tabular}{|l|l|l|l|}
\hline & KPC & NDM & OXA-48 \\
\hline Molecular class & A & B & D \\
\hline Common species & K. pneumoniae & $\begin{array}{l}\text { K. pneumoniae } \\
\text { E. coli }\end{array}$ & K. pneumoniae \\
\hline $\begin{array}{l}\text { Regions/countries with high } \\
\text { burden }\end{array}$ & $\begin{array}{l}\text { United States } \\
\text { Brazil } \\
\text { Italy } \\
\text { Greece } \\
\text { Israel } \\
\text { China }\end{array}$ & $\begin{array}{l}\text { South Asia (India, Pakistan, } \\
\text { Bangladesh, Nepal) }\end{array}$ & $\begin{array}{l}\text { Mediterranean (Turkey, Algeria, } \\
\text { Lebanon, Libya, Tunisia, Morocco) } \\
\text { Gulf (Saudi Arabia) }\end{array}$ \\
\hline Spectrum of resistance & $\begin{array}{l}\text { All } \beta \text {-lactams including } \\
\text { carbapenems }\end{array}$ & $\begin{array}{l}\text { All } \beta \text {-lactams including } \\
\text { carbapenems but except } \\
\text { aztreonam }\end{array}$ & Penicillins and carbapenems \\
\hline $\begin{array}{l}\text { Inhibited by classic } \beta \text {-lactamase } \\
\text { inhibitors }\end{array}$ & Minimally & No & No \\
\hline Inhibited by avibactam & Yes & No & Yes \\
\hline
\end{tabular}

